
allemande

\title{
Les Lauensteiner Kulturtagungen de 1917 : «l'extraordinaire discordance des points de vue »
}

Nepthys Zwer

\section{(2) OpenEdition}

1 Journals

Édition électronique

URL : https://journals.openedition.org/allemagne/572

DOI : 10.4000/allemagne.572

ISSN : 2605-7913

Éditeur

Société d'études allemandes

Édition imprimée

Date de publication : 29 décembre 2017

Pagination : 301-313

ISSN : 0035-0974

\section{Référence électronique}

Nepthys Zwer, «Les Lauensteiner Kulturtagungen de 1917 : « l'extraordinaire discordance des points de vue » », Revue d'Allemagne et des pays de langue allemande [En ligne], 49-2 | 2017, mis en ligne le 29 décembre 2018, consulté le 18 mai 2021. URL : http://journals.openedition.org/allemagne/572 ; DOI : https://doi.org/10.4000/allemagne.572 


\section{Les Lauensteiner Kulturtagungen de 1917: «l'extraordinaire discordance des points de vue»}

- Nepthys Zwer*

Au printemps et à l'automne 1917, l'éditeur Eugen Diederichs (1867-1930) organisait deux rencontres culturelles à Lauenstein, un petit château fort près de Probstzella, au cœur des monts de Thuringe de Haute-Franconie. Les exposés et les discussions passionnées qui ont animé ces journées portaient sur le renouveau de l'Allemagne à l'issue de la guerre, à un moment où «l'esprit de 1914 » avait commencé à s'éroder et où l'on pouvait entrevoir la future démocratisation du Reich ${ }^{(1)}$.

Bien qu'elles aient réuni de nombreux intellectuels, ces rencontres ont peu été commentées. En effet, pour permettre à chacun de s'exprimer librement en dépit de la censure, les organisateurs et leurs hôtes avaient convenu de ne publier aucun compte rendu officiel des interventions et débats. Pour évoquer ces rencontres, le présent article peut cependant s'appuyer sur un protocole interne rédigé par Wolfgang Schumann ${ }^{(2)}$, sur la correspondance et les mémoires d'Eugen Diederichs, ainsi que sur de courtes évocations dans des articles de la revue Die Tat.

* Docteur en études germaniques.

1 Sur les développements géopolitiques et idéologiques de «l’année de rupture» 1917, voir Jean-Yves LE Naour, 1917. La paix impossible, Paris, Perrin, 2015; sur les événements, voir Wolfdieter BiHL, Der Erste Weltkrieg. 1914-1918, Vienne, Böhlau, 2010, p. 154-209.

2 Wolfgang Schumann, «Darstellung der Haupttendenzen, welche auf der Lauensteiner Tagung der Vaterländischen Gesellschaft zu Tage traten», protocole de la première rencontre, reproduit dans Gangolf HüBINGer, «Eugen Diederichs Bemühungen um die Grundlegung einer neuen Geisteskultur», in: Wolfgang Mommsen (éd.), Kultur und Krieg: Die Rolle der Intellektuellen, Künstler und Schriftsteller im Ersten Weltkrieg, Munich, Oldenbourg, 1996, p. 259-274, annexes p. 268-274. Le journaliste culturel Wolfgang Schumann (1887-1964), beau-fils de Ferdinand Avenarius, est le secrétaire du Dürerbund, co-organisateur des rencontres. 
Max Weber et Ernst Toller parmi un groupe d'invités, Lauensteiner Tagung de l'automne 1917

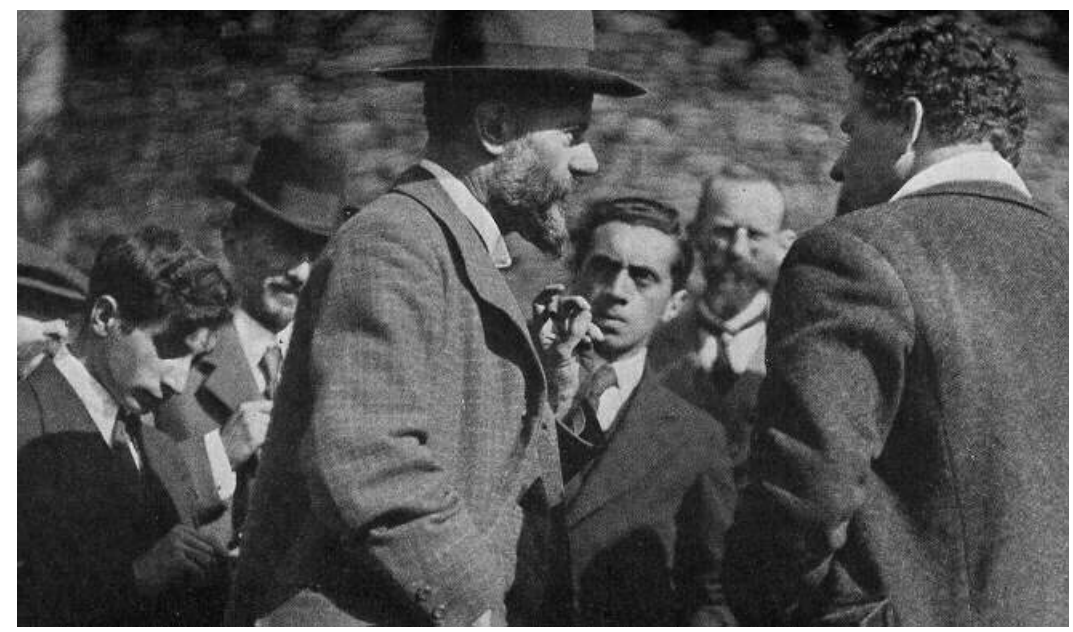

Source: http://web.archive.org/web/20070312115641/http://www.staff.uni-marburg.de/ $\sim$ kaesler/max.html.

L'image que l'histoire a retenue de ces rencontres est celle du dramaturge Ernst Toller (1893-1939) levant sur Max Weber (1864-1920) des yeux scrutateurs. Cet instant, pris sur le vif, témoigne de «l'extraordinaire discordance des points de vue » ${ }^{(3)}$ qui s'est faite jour à Lauenstein, divergences que Diederichs avait espéré voir dépassées, mais qui ne firent que se cristalliser au fil des rencontres et qui marquèrent les esprits de nombreux participants, comme nous allons le voir.

\section{Des académies platoniciennes au château}

Dans la biographie de son mari, Marianne Weber évoque sur plusieurs pages les rencontres de Lauenstein. Elle en campe ainsi le décor:

"C'est un lieu plein de charme. Dans les pièces meublées à l'ancienne les fenêtres s'ouvrent sur les pentes boisées des collines et le regard s'enfonce dans les replis des vallées alentours - les gens se retrouvent dans la salle d'armes ou dans la cour du château. - Les pampres colorés de la vigne sauvage recouvrent les murs. Du pignon pend le drapeau allemand - le symbole d'une communauté supra personnelle. Tout ce qui, dans ce cadre historique, a défié l'assaut du temps, rappelle aussi les racines communes d'une culture allemande, d'un passé où chaque chose et chaque être avait sa place assignée, bien à l'abri au sein d'un ordre bien compris» ${ }^{(4)}$.

Le château, en réalité, a été entièrement reconstruit par un amateur qui l’a transformé en lieu de congrès et en hôtel. L'atmosphère médiévale savamment reconstituée suggère une immersion dans le passé germanique et les sombres forêts de sapins

3 W. Schumann, ibid., p. 273-274.

4 Marianne Weber, Max Weber. Ein Lebensbild (1926), Tübingen, Mohr Siebeck, 1984, p. 609. Toutes traductions N.Z. 
alentours invitent à de longues promenades. Dans cet endroit propice à la réflexion, Diederichs se plaît à imaginer un "genre d'académie platonicienne», comme il le suggère à Max Weber dans sa lettre d'invitation ${ }^{(5)}$.

En choisissant ce cadre sylvestre et médiévisant pour ses rencontres, Eugen Diederichs ne sacrifie pas uniquement au folklore pangermaniste. L'histoire a certes retenu de lui son évolution vers l'idéologie völkisch dans l'entre-deux-guerres, mais, en 1917, il défend encore des idées démocratiques et internationalistes. Tout comme Max Weber, il dénonce même avec véhémence le «danger alldeutsch».

Le Kulturverlag Diederichs, fondé en 1896, édite à Iéna des ouvrages reflétant, selon son fondateur, les «tendances modernes» de la littérature et des sciences. Diederichs publie aussi la revue Die Tat, conçue comme un forum d'expression et de discussion ouvert aux avis contradictoires. L'éditeur est persuadé que de la confrontation des idées naîtront des impulsions productives sur le «front intérieur» (Heimatfront), celui de la vie culturelle. Pour Diederichs, l'édition a pour mission d'éduquer le peuple et lui-même s'imagine en passeur et organisateur de cette éducation. Faisant fi de la rentabilité de son entreprise (et de la censure), il veut générer un état d'esprit «éthique» qui inaugurerait un nouvel idéalisme. C'est là l'enjeu de la Kulturfrage: impliquer davantage «l'esprit» dans la vie économique et politique. Pour restituer à l'Allemagne sa dimension spirituelle, les esprits créatifs doivent donc s'impliquer politiquement. L'éditeur compte pour cela sur les acteurs de la Lebensreform, mais aussi sur ceux des mouvements de jeunesse et des ligues culturelles (Kulturbünde) et c'est à cette fin qu'il leur offre une tribune dans ses publications ${ }^{(6)}$. Organiser des «rencontres culturelles» lui semble un autre moyen de parvenir à ses fins.

Avec Lauenstein, Diederichs réalise donc un projet qui lui tient à cœur : faire se rencontrer et se confronter des intellectuels - des penseurs, des personnalités morales, des créatifs - qui initieront une dynamique de renouvellement de l'Allemagne. Cette nouvelle Allemagne doit prendre conscience de son identité particulière et du rôle culturel qu'elle est amenée à jouer auprès des autres nations ${ }^{(7)}$. Diederichs exprime aussi un désir que partagent beaucoup de ses invités: la volonté de commuer l'expérience de la guerre en un acte positif de reconstruction, de transformer «le chaos en création ${ }^{(8)}$. Ce sont ces volontés qu'il se propose de coaliser, se demandant toutefois s'il parviendra à rallier la jeunesse ${ }^{(9)}$. En effet, le Freideutscher Jugendtag, qui s'était tenu sur la montagne du Hoher Meißner en 1913, l'avait déçu dans la mesure où il n'y avait pas trouvé cette jeunesse, ferment du renouveau, qu’il espérait.

5 Lettre d'invitation d'Eugen Diederichs à Max Weber du 24 mai 1917, HS.1995.0002, archives de la maison d'édition Eugen Diederichs, Deutsches Literaturarchiv, Marbach am Neckar (ci-après DLA).

6 Sur l'histoire de la maison d'édition, voir Irmgard Heidler, Der Verleger Eugen Diederichs und seine Welt (1896-1930), Wiesbaden, Harrassowitz, 1998, p. 399 sq.

7 «Organisation des deutschen Kultureinflußes im Ausland». Eugen Diederichs, feuille de calepin non datée, HS.1995.0002, archives de la maison d'édition Eugen Diederichs, DLA.

8 «Das Chaos umbiegen in Schöpfung». Max Maurenbrecher, «Der Krieg als Ausgangspunkt einer deutschen Kultur», Die Tat. Monatschrift für die Zukunft deutscher Kultur, n 2 (mai 1917), p. 97-107, ici p. 101.

9 Lettre d'Eugen Diederichs à Otto Crusius du 27 février 1917, reproduite dans Lulu von STRAuss und Torney-Diederichs (éd.), Eugen Diederichs. Leben und Werk. Ausgewählte Briefe und Aufzeichnungen, Iéna, Eugen Diederichs Verlag, 1936, p. 288-290, ici p. 290. 
Son ambition première est de donner aux rencontres de Lauenstein les dimensions d'un véritable congrès qui débattrait de la politique du moment. Cependant, les contraintes de la guerre, tant organisationnelles que politiques, l'obligent à réviser ses ambitions à la baisse pour se contenter d'une rencontre plus «intime». Ces «discussions à huis clos» entre gens d'esprit doivent permettre aux participants de mieux se connaître, de partager «un moment de vie commune» dans une «belle nature» ${ }^{(10)}$.

Les invitations portent la signature de trois organismes: Diederichs signe pour la Vaterländische Gesellschaft für Thüringen 1914 (qu'il a cofondée avec Max Maurenbrecher et Wilhelm Vershofen), Ferdinand Jakob Schmidt pour la Comenius-Gesellschaft et Ferdinand Avenarius pour le Dürerbund. Les participants doivent s'acquitter d'une petite contribution financière pour l'hébergement, les repas et les prestations artistiques et sont priés de ne pas oublier leurs cartes de rationnement. La plupart seront logés au village (ou devront organiser leur propre hébergement), le château ne disposant que de 15 chambres. Les dames ne sont admises qu'en tant qu'accompagnatrices et, ce, à condition qu'il reste de la place.

Les deux rencontres se déroulent de façon semblable: des exposés sur un thème donné (arrêté après consultation préalable des personnes intéressées) alternent avec des débats tout au long de la journée, certaines soirées étant agrémentées de prestations artistiques, tel ce mystère médiéval tenu dans la cour du château. Diederichs tient d'ailleurs beaucoup à offrir à ses hôtes ces «événements culturels».

\section{L'avenir de l'Allemagne}

La première rencontre, la Pfingsttagung, se tient à la Pentecôte, du 29 au 31 mai 1917, et réunit une soixantaine d'invités - des politiciens, des juristes, des professeurs, des enseignants, des médecins, des écrivains, des artistes et des étudiants, toutes couleurs politiques confondues ${ }^{(11)}$.

Le thème proposé pour cette première rencontre, «Sens et devoir de notre époque» («Sinn und Aufgabe unserer Zeit»), doit traiter de questions de politique intérieure

10 Carton d'invitation pour la première rencontre, HS.1995.0002, archives de la maison d'édition Eugen Diederichs, DLA.

11 Dans son compte rendu, Wolfgang Schumann évoque les personnes suivantes: Eugen Diederichs et son épouse, Max Maurenbrecher, Max Weber, Hermann Barge, professeur de lycée à Leipzig, Paul Lensch, député SPD au Reichstag, Franz Staudinger, professeur de lycée à Darmstadt, du mouvement coopératif (Konsumgenossenschaftsbewegung), SPD, Gerhard Seeliger, historien à l'université de Leipzig, Wilhelm von Blume, juriste à l'université de Tübingen, Eduard Wechßler, professeur de littérature à l'université de Marbourg, Theodor Heuss, rédacteur du journal linksbürgerlich «März», Knud Ahlborn, médecin à Munich, rédacteur en chef du journal Freideutsche Jugend, Harald Schultz-Henke, médecin, Erich Trummler, étudiant, Wolfgang Wallach, étudiant, Kurt Kroner, plasticien, Carl Emil Uphoff, peintre, membre de la Worpsweder Künstlerkolonie, Wilhelm Vershofen, poète, économiste, Hans Kampffmeyer, paysagiste, président de la Deutsche Gartenstadtbewegung, Karlsruhe, Ferdinand Jacob Schmidt, enseignant à l'université de Berlin, président de la Comenius-Gesellschaft, Richard Dehmel, poète, près de Hambourg, Werner Mahrholz, journaliste à Munich, plus tard rédacteur politique de la Vossische Zeitung (certaines précisions biographiques sont apportées par Gangolf Hübinger). Le carton d'invitation pour la deuxième rencontre désigne également comme ayant participé à la première rencontre d'autres personnes, probablement restées discrètes, notamment l'historien Friedrich Meinecke, le sociologue Alfred Vierkandt et l'écrivain Walter von Molo. 
(réforme du droit de vote, réforme fiscale, question sociale, éducation) et extérieure. De fait, les discussions tourneront plutôt autour d'une définition de l'État (et de sa conception spécifiquement germanique) et du rôle et de la place de l'Allemagne parmi les nations. Les hôtes ont d'ailleurs été invités à prendre au préalable connaissance de l'ouvrage fraîchement paru de Walther Rathenau, Von kommenden Dingen ${ }^{(12)}$. Celui-ci traite la question du renouveau de l'Allemagne sur un plan très théorique: contre la «mécanisation " générale de la vie, l'auteur prône un idéal de morale, de responsabilité et de solidarité qui se concrétiserait dans un Volksstaat sans classes ni privilèges.

Pour inaugurer la première journée, l'éditeur a fait appel au théologien Max Maurenbrecher (1874-1930). Maurenbrecher espère, tout comme lui, que Lauenstein provoquera « une stimulation, une unification et un approfondissement de la façon de penser des participants eux-mêmes et ainsi, avec le temps, peut-être aussi un effet dans tous les domaines de la vie intellectuelle et publique allemande» ${ }^{(13)}$. Les deux hommes sont convaincus que l'élite intellectuelle du pays est appelée à jouer un rôle important dans la future Allemagne et nombreux sont les participants qui s'imaginent pouvoir «gagner les âmes " par leurs discours. En réalité, Diederichs a commencé à se distancier des idées du conservateur révolutionnaire avec qui il avait un temps imaginé inventer une nouvelle «religiosité» (Maurenbrecher entrera à la Deutsche Vaterlandspartei à l'été 1917 et leur rupture sera consommée après la deuxième rencontre). Mais, en endossant le rôle de la vieille garde nationaliste et fidèle au Kaiser, celui-ci doit provoquer la réaction des convives et permettre d'engager de fructueuses discussions. En cela, Diederichs ne se sera pas trompé: les réactions seront nombreuses et parfois violentes.

Le discours d'ouverture de Max Maurenbrecher, "Über die deutsche Staatsidee», reprend les idées de son article «Der Krieg als Ausgangspunkt einer deutschen Kultur» (14), qui propose de "réaliser une culture authentiquement allemande bien que tournée vers l'avenir et l'éternel devenir». Il oppose la spiritualité et le sens de la communauté spécifiquement germaniques (ce qu'il entend par «Volkstum») à l'individualisme démocratique de l'Europe de l'Ouest. Le libéralisme et le capitalisme «mécanicistes» lui semblent les conséquences du rationalisme. Se référant aux classiques allemands, il rêve d'un État fort, à la "prussienne»: l'État est le principe premier duquel émergent le peuple et la nation. Une prise de conscience - également dans le monde ouvrier - permettrait de forger cette nouvelle volonté générale. Et, pour guider les Allemands sur cette voie rédemptrice, il imagine même créer un «parti des intellectuels ».

Son exposé, long de quatre heures, soulève de vives réactions et les discussions se poursuivent jusqu'à trois heures du matin. Il en sera ainsi pendant tout le congrès: du mardi soir au vendredi midi les interventions suscitent prises de partie et oppositions. C'est surtout le «romantisme d'État» et le nationalisme de Maurenbrecher qui se voient vilipendés par la majorité des auditeurs. Par contre, la lutte à mener contre le capitalisme fait l'unanimité, même si la mise en avant de la primauté de l'intérêt général correspond moins à un souci de démocratie que de la (re)valorisation de

12 Walther Rathenau, Von kommenden Dingen (1917), Berlin, Fischer, 1918.

13 M. Maurenbrecher, «Der Krieg als Ausgangspunkt einer deutschen Kultur» (note 8), p. 100.

14 Ibid., p. 100-101. 
l'«esprit de communauté»(Gemeinschaftsgeist). Contre le capitalisme, chacun propose ses propres solutions: le socialisme, le mouvement coopératif ou l'instauration d'une culture politique digne de ce nom, comme le fait Max Weber. Pour celui-ci la «mécanisation du monde» et la bureaucratie sont inévitables, mais l'instauration d'un véritable jeu politique, qui aboutira à la sélection naturelle des leaders les plus compétents, est une panacée pour les maux dont souffre le Reich ${ }^{(15)}$.

L'intervention de Weber est brillante et attise les esprits. Wolfgang Schumann constate la «fracture» qu'elle instaure dans les discussions. De fil en aiguille, on en vient à la question de la guerre et de la responsabilité de l'Allemagne (Kriegsschuldfrage). Alors que les intervenants plus âgés se sont cantonnés à des considérations philosophiques, ce sont les jeunes qui posent la question. Ces jeunes - des représentants des jeunesses universitaires, des étudiants, des artistes - reviennent tout juste des champs de bataille et refusent de mythifier cette guerre. Elle ne leur semble pas un affrontement entre culture allemande et civilisation occidentale ou entre des intérêts capitalistes divergents, mais une pure folie («Wahnsinn»), due au manque de valeurs morales de tous les belligérants confondus. Ils ne peuvent lui trouver un quelconque sens historique. Pour le jeune médecin Knud Ahlborn (1888-1977), président de la Deutsche Akademische Freischar et de la Freideutsche Jugend (la fédération de toutes les ligues de jeunesse présentes au Hoher Meißner), il faut parvenir à la moralisation et à la responsabilisation de la vie publique. Pour ceci, les jeunes participants proposent l'abstinence à l'alcool, l'éducation, le refus du luxe, la réforme agraire, le projet coopératif, les cités-jardins, mais aussi, de façon moins prosaïque et plus confuse, la création d'une nouvelle religion.

Weber a du mal à comprendre le point de vue de la «jeunesse», qu'il taxe d'ésotérisme, et, face au sarcasme de ses attaques, une partie des jeunes hôtes finit par se détourner de lui ${ }^{(16)}$. C'est ici que, dans son compte rendu, Wolfgang Schumann fait état d'une deuxième fracture, non plus idéologique mais générationnelle. Diederichs, qui avait espéré que la jeunesse répondrait à son appel, s'enthousiasme, certes, pour ces jeunes si motivés, avec lesquels il imagine enfin possible de créer une sorte de communautés de cœur et d'esprit ${ }^{(17)}$. Mais, parmi eux, beaucoup refusent de faire si promptement l'économie du drame qu'ils viennent de vivre et n'envisagent aucunement de rallier le mysticisme quelque peu élitiste de Diederichs. Ils affirmeront leurs positions lors de la rencontre suivante.

15 Pour Max Weber, le «Redeparlament», le "parlement de la parlotte» actuel, est corrompu par les prises d'intérêts personnelles et ne remplit pas son office. Voir le compte rendu de Wolfgang Schumann, p. 270-271. Voir également les écrits et discours de Max Weber dans Wolfgang J. Mommsen et Gangolf Hübinger (éd.), Max Weber. Zur Politik im Weltkrieg. Schriften und Reden 1914-1918, Tübingen, Mohr Siebeck, 1984; également Wolfgang J. Mommsen et Wolfgang Schwentker (éd.), Max Weber. Zur Neuordnung Deutschlands. Schriften und Reden 1918-1920, Tübingen, Mohr Siebeck, 1988; aussi Joachim Radkau, Max Weber. Die Leidenschaft des Denkens, Munich, Carl Hanser Verlag, 2005. Weber imposera le thème de la Führerauslese, de la sélection des leaders, pour la deuxième rencontre. 


\section{Une jeunesse en quête de clarté}

La deuxième rencontre se tient du 29 septembre au 3 octobre 1917 et porte sur la question du «guide» dans l'État et la culture («Das Führerproblem im Staate und in der Kultur»). Il s'agit de traiter de «la question du développement de l'État allemand et de la position future des individus et des groupes politiques à l'intérieur et face à l'État, au regard de nos tâches à venir et des derniers événements de politique intérieure ${ }^{(18)}$.

Les organisateurs réinvitent les mêmes personnes dans l'idée de les laisser poursuivre et approfondir leurs propos, mais l'on compte aussi de nouveaux hôtes, entre autres le sociologue Ferdinand Tönnies, l'économiste Werner Sombart, la féministe Gertrud Bäumer et le philologue Otto Crusius. L'anarchiste pacifiste Gustav Landauer, lui, s'est désisté. Les intervenants sont, entre autres, Max Weber, Max Maurenbrecher, Ferdinand Tönnies, le pédagogue Ernst Krieck, le juriste Karl Polenske, l'enseignant Curt Heinke et Otto Neurath.

L'exposé de Max Weber, «La personnalité et l'ordre de vie» («Die Persönlichkeit und die Lebensordnung»), ouvre les débats. La sélection des leaders, l'économie et la «question religieuse» sont également au programme. "Foi, amour et espoir» note Ferdinand Tönnies dans son calepin à propos de son propre exposé(19).

Le jeune économiste autrichien Otto Neurath ${ }^{(20)}$, avec un exposé intitulé «L'ordre économique à venir» ${ }^{(21)}$, donne aux débats une dimension plus pragmatique qui s'inscrit dans la préoccupation du moment pour la conduite de l'économie de l'aprèsguerre. La Gemeinwirtschaft, l'économie coopérative et sociale, a, depuis les années 1916, de nombreux adeptes ${ }^{(22)}$. Afin de sortir de l'impasse induite par la pratique du "laissez-faire» de l'économie de marché depuis 200 ans, Neurath propose de maintenir l'économie planifiée de la guerre (Kriegswirtschaft), avec un interventionnisme étatique «énergique» (suppression des intermédiaires de commerce, prix fixes, échanges en nature, etc.). Cette refondation radicale du fonctionnement de l'économie doit se faire sur des bases scientifiques: les experts de l'économie et de l'organisation sociale, les Gesellschaftstechniker, doivent concevoir des plans alternatifs et les soumettre à l'appréciation générale ${ }^{(23)}$. C'est en 1917 que Neurath élabore ce système des

18 Carton d'invitation pour la deuxième rencontre, HS.1995.0002, archives de la maison d'édition Eugen Diederichs, DLA.

19 Les notes que Ferdinand Tönnies consigne dans son journal n'apportent malheureusement aucune précision sur les débats. Voir leur reproduction dans Mommsen/Hü BInger (éd.), Max Weber (note 15), p. 707.

20 La présence d'Otto Neurath en tant que simple auditeur lors de la première rencontre n'est pas attestée, mais tout à fait imaginable en raison de ses liens d'amitié avec Wolfgang Schumann.

21 Voir la série de trois articles qu'il publie alors sur ce thème: Otto NeURATH, «Die Wirtschaftsordnung der Zukunft und die Wirtschaftswissenschaften ", Der Österreischiche Volkswirt, 1917, p. 501-505, 521524, 544-548; texte également publié au Verlag für Fachliteratur de Berlin en 1917.

22 Dès 1917, la maison d'édition de Diederichs, qui s'est largement ouverte aux écrits politiques, abrite la collection «Deutsche Gemeinwirtschaft», qui comptera 18 publications jusqu'en 1920. On y trouve des écrits de Wichard von Moellendorff, Rudolf Wissell, Erich Schairer, Walther Rathenau et Otto Neurath.

23 Dès 1917, Neurath s'inscrit, sans le savoir, dans le mouvement plus large de l'ingénierie sociale telle qu'elle s'est manifestée en Occident au début du $\mathrm{XX}^{\mathrm{e}}$ siècle. Voir Nepthys ZwEr, L'ingénierie sociale 
«constructions technico-sociales» pour lequel il inventera également le terme d'«utopistique scientifique» ${ }^{(24)}$.

La «jeunesse» présente à Lauenstein cherche indubitablement à prendre en main son propre avenir. Pour cela, elle a besoin d'un guide. Ainsi en est-il du tout jeune étudiant Ernst Toller ${ }^{(25)}$, engagé volontaire en 1914, revenu meurtri physiquement et psychiquement des tranchées. Dans son autobiographie, il consacrera un long passage à Lauenstein, qui se révèle une expérience décisive pour son parcours politique ${ }^{(26)}$. Toller a compris l'initiative de Diederichs comme une tentative de remédier à la perte générale de repères en ces temps troubles, où «tous doutent des valeurs d'hier et d'aujourd'hui ». Il fait partie de «ceux qui cherchent » ${ }^{(27)}$ un guide spirituel qui les sortirait de la confusion.

Max Weber, par sa probité intellectuelle et son franc-parler ${ }^{(28)}$, semble, de prime abord, pouvoir assumer ce rôle et «la jeunesse se raccroche» à lui. Pourtant, il la déçoit. C'est que le " professeur » œuvre à retrouver la grandeur de l'Allemagne et ne remet pas en question le rôle de cet État et son pouvoir. Les jeunes, eux, veulent «autre chose que de réformer seulement le droit de vote, ils veulent bâtir de nouvelles fondations, ils croient que la transformation de l'ordre extérieur transforme aussi l'être humain » ${ }^{(29)}$. À cela, la quête spirituelle de Diederichs et ses «professions de foi » ne suffisent plus.

Entre les deux rencontres, l'état d'esprit dans les tranchées a changé et l'on voit se multiplier les actes de protestations de soldats allemands remettant en question les ordres de leurs supérieurs ${ }^{(30)}$. Pour les jeunes de Lauenstein, l'expérience de la guerre a ouvert une brèche insurmontable entre les générations : ces pères qui «ne se sont jamais retrouvés couchés dans les tranchées» ont perdu tout crédit aux yeux de leurs enfants. Pour ces derniers, la guerre n'est pas une fatalité, le «sort réservé à l'Allemagne par son dieu ${ }^{(31)}$ ou une déception politique, elle est un drame humain imputable à la génération précédente. Selon Ernst Toller, «seuls les jeunes veulent la clarté» et «le verbiage,

d'Otto Neurath, à paraître aux Presses universitaires de Rouen et du Havre en 2017; sur le concept d’ingénierie sociale, voir Thomas Etzemüller (éd.), Die Ordnung der Moderne. Social Engineering im 20. Jahrhundert, Bielefeld, transcript Verlag, 2009.

24 Voir Otto Neurath, "Die Utopie als gesellschaftstechnische Konstruktion», in: Otto Neurath, Durch die Kriegswirtschaft zur Naturalwirtschaft, Munich, Callwey, 1919, p. 228-231.

25 La présence d'Ernst Toller à la première rencontre n'est aucunement avérée, malgré une affirmation fréquemment reprise par les historiens, probablement en raison de sa propre formulation imprécise dans son autobiographie, Eine Jugend in Deutschland (1933), Stuttgart, Reclam, 2011, p. 467.

26 E. Toller, Eine Jugend in Deutschland, ibid., p. 77-85. Les souvenirs de Toller sont fortement idéalisés.

27 Lettre de Wolfgang Schumann à Eugen Diederichs du 11 octobre 1917, HS.1995.0002, archives de la maison d'édition Eugen Diederichs, DLA.

28 Weber déclare vouloir humilier le Kaiser et les responsables politiques pour qu'ils passent aux aveux à propos du déclenchement de la guerre. Cela donne, bien sûr, des sueurs froides aux autres invités. Voir Theodor Heuss, Erinnerungen 1905-1933, Tübingen, Rainer Wunderlich Verlag, 1963, p. 215.

29 E. Toller, Eine Jugend in Deutschland (note 25), p. 79.

30 Sur l'esprit de révolte des soldats allemands, voir Anne Lipp, Meinungslenkung im Krieg. Kriegserfahrungen deutscher Soldaten und ihre Deutung 1914-1918, Göttingen, Vandenhoeck \& Ruprecht, 2003, p. 139-147 et p. 289-296.

31 «Der Krieg ist eine Schickung des deutschen Gottes [...]». Max Maurenbrecher cité par E. ToLLER, Eine Jugend in Deutschland (note 25), p. 77-78. 
l'inaction, la lâcheté» les dégoûtent. Il quittera Lauenstein profondément déçu et se souviendra: «De grandes paroles furent prononcées, mais rien ne s'est passé » ${ }^{(32)}$.

Lors de cette deuxième rencontre, l'esprit constructif semble, de toute manière, s'être délité et Wolfgang Schumann tente en vain de le ranimer. Il déplore surtout les agissements d'un cercle d'écrivains autour de Wilhelm Vershofen (groupe qu'il nomme la «Quadriga» en référence à sa revue $\left.{ }^{(33)}\right)$, qui profite de Lauenstein pour organiser des entrevues privées, excluant ainsi les autres participants des discussions et les condamnant à «rester assis là », «à s'ennuyer " ${ }^{(34)}$.

De plus, le programme culturel ne fait pas l'unanimité. L'épouse d'Eugen Diederichs, l'écrivain Lulu von Strauß und Torney (1873-1956), et le poète Karl Bröger (1886-1944) déclament des poèmes. Les filles de l'écrivain Gustav Falke dansent au clair de lune - prestation fort critiquée par nombre d'invités en raison de leur amateurisme avéré. Selon Ernst Toller, «que ce soit bon ou mauvais, peu importe, les professeurs croient que l'esprit de dieu plane au-dessus de cette danse. Quand ils déambulent dans les appartements aux lambris sombres, dans lesquels de vieux meubles véreux mènent leur existence fantomatique, ils se prennent pour des chevaliers médiévaux, pour des missionnaires de l'Esprit saint ${ }^{(35)}$.

Ces rencontres, qui semblaient donc aux uns une expérience authentique, se révélaient pour les autres une «tromperie», comme ce fut le cas pour Max Weber ${ }^{(36)}$.

\section{Lauenstein et la suite}

Pourtant, malgré les incidents et les différends, les Lauensteiner Tagungen ont paru une réussite à leurs organisateurs. Wolfgang Schumann se déclara "presque tout à fait satisfait» et voulut réitérer l'expérience. Aux yeux de Diederichs, leur succès tenait avant tout à la présence de Max Weber, cet «esprit dominant» («überragender Geist») ${ }^{(37)}$, qu'il considérait comme sa «plus belle acquisition» ${ }^{(38)}$. L'éditeur s'enorgueillit d'être parvenu à réunir «des savants, des artistes et des praticiens » et de leur faire vivre, malgré leurs dissensions, une entreprise spirituelle commune. Son idée fit même des adeptes, on chercha à l'imiter et se proposa de l'aider à préparer la prochaine rencontre. Un collège spontané de six hommes (Sechs-Männer-Kollegium) composé

32 Ibid., p. 80.

33 La revue Quadriga était éditée par le groupe d'artistes «Werkleute auf Haus Nyland» dans la mouvance du Werkbund et dont faisait partie l'économiste et écrivain Wilhelm Vershofen (1878-1960). Les poètes Richard Dehmel et Karl Bröger, présents à Lauenstein, en étaient également membres.

34 Voir la lettre de Wolfgang Schumann à Eugen Diederichs du 11 octobre 1917, HS.1995.0002, archives de la maison d'édition Eugen Diederichs, DLA.

35 E. Toller, Eine Jugend in Deutschland (note 25), p. 78.

36 «Die Sache selbst ist Schwindel (Diederichs'sche Verleger-Angelegenheit!), aber die Situation höchst pittoresk und nette Menschen da. " Lettre de Max Weber à Ludo Moritz Hartmann du 8 septembre 1917, reproduite dans Gerd KRUMreich et Rainer Lepsuis (éd.), Max Weber: Briefe 1915-1917, Tübingen, Mohr Siebeck, 2008, p. 771-772, ici p. 772.

37 Eugen Diederichs, «Vom Wesen einer Kulturtagung», Die Tat, nº 11 (février 1918), p. 950-952, ici p. 952.

38 Lettre d'Eugen Diederichs à Friedrich von der Leyen du 6 octobre 1917, reproduite dans L. von Strauss und Torney-Diederichs (éd.), Eugen Diederichs (note 9), p. 302. 
d'Eugen Diederichs, Max Maurenbrecher, Wolfgang Schumann, Max Weber, Wilhelm Vershofen et Otto Neurath s'attela aux préparatifs. Ils décidèrent d'un système de sélection des invitations, chaque nom proposé par l'un d'entre eux étant soumis au veto inappelable des cinq autres ${ }^{(39)}$. Les thèmes retenus pour la future rencontre furent la Jugendbewegung, le pacifisme, le prolétariat et la social-démocratie. Toutefois, Wolfgang Schumann exigea qu'on évite, à l'avenir, une certaine mièvrerie « historisante»...

La période troublée de la fin de la guerre aura raison du projet. Les participants durent se dédire en masse, à commencer par le collège des six lui-même. Dès le début, Lulu von Strauß und Torney interféra dans l'organisation, tenant à inviter, au grand dam de Wolfgang Schumann, de simples étudiants. Ceci permit cependant d'organiser, en dépit des désistements, une réunion, plus informelle, entre jeunes socialistes, notamment avec la Freideutsche Jugend et des poètes ouvriers ${ }^{(40)}$.

Diederichs reprendra le principe un peu plus tard, organisant à Lauenstein des rencontres d'éditeurs dans le même esprit ${ }^{(41)}$. Ce type de rencontres culturelles satisfaisait en somme aux attentes des organisateurs et de beaucoup de participants.

Lauenstein permit à Max Weber de se rapprocher de la jeunesse. C'est là qu'il accepta la proposition des Freistudenten d'organiser la conférence «Wissenschaft als Beruf» qu'il tiendra à Munich le 7 novembre 1917, soit un mois après la deuxième rencontre. Lors de sa conférence «Politik als Beruf», donnée le 28 janvier 1919 pour ces mêmes étudiants, il développera son idéal de l'homme politique ${ }^{(42)}$. Il ouvrira aussi les portes de sa maison de Heidelberg à cette jeunesse en quête d'orientation.

Il faut dire que l'université de Heidelberg a la réputation d'être une «usine à docteurs » et attire nombre d'étudiants. Otto Neurath y est habilité dans le courant de l'été 1917. À l'automne 1917, Ernst Toller s'y inscrit en droit et il est alors admis au cercle intime des étudiants socialistes et pacifistes de la maison Weber. Devenu "rebelle», selon ses propres termes, il s'engage alors dans l'activisme politique et prend contact avec l'anarchiste Gustav Landauer. Mais c'est à Berlin, en janvier 1918, qu'il rencontrera, en la personne du Arbeiterführer Kurt Eisner, le guide tant espéré qui l'entraînera sur le chemin de la révolution bavaroise. Il y retrouvera d'autres jeunes participants de Lauenstein, tels Wolfgang Schumann et Otto Neurath, également happés par le tourbillon de la «République des Conseils» de Munich au printemps $1919^{(43)}$. On devine un discret fil conducteur qui relie Lauenstein à la révolution bavaroise et qui passe, à son corps défendant, par la maison Weber.

À l'été 1919, quand Max Weber sera appelé à témoigner aux procès d'Ernst Toller et d'Otto Neurath, accusés de haute trahison (de complicité pour le second), il arguera

39 Lettre de Wolfgang Schumann à Eugen Diederichs du 3 février 1917, HS.1995.0002, archives de la maison d'édition Eugen Diederichs, DLA.

40 Voir I. Heidler, Der Verleger Eugen Diederichs (note 6), p. 98.

41 Ces rencontres de l'édition (Buchhandelstagungen) se sont tenues de 1922 à 1926. Voir ibid., p. 117-129.

42 Voir la transcription de ces textes dans Max Weber, Wissenschaft als Beruf, 1917/1919; Politik als Beruf, 1919 (1919), Tübingen, Mohr Siebeck, 1994.

43 Wolfgang Schumann et Otto Neurath avaient conçu dans un premier temps, avec l'aide du journaliste Hermann Kranold, la Kranold-Neurath-Schumann-Denkschrift, censée révolutionner le système économique de la Saxe. Hermann Kranold, rédacteur de la Chemnitzer Volksstimme, invité à la $3^{\mathrm{e}}$ rencontre, s'était désisté. 
pour les défendre de leur immaturité et de leur aveuglement ${ }^{(44)}$. Pour défendre Toller, il n'hésitera pas à invoquer l'effet «tordu» des rencontres de Lauenstein ${ }^{(45)}$. Le «mythe de Heidelberg» mourra prématurément en 1920, prêt à s'engager dans la transformation républicaine du Reich. En décembre 1918, à la demande de Hugo Preuß, il avait contribué à la mise sur pied du projet de constitution allemande.

Après l'échec de la révolution bavaroise, écrasée dans le sang, Otto Neurath retournera à Vienne - devenue Vienne-la-Rouge - et s'engagera auprès des austromarxistes dans le renouvellement de l'ordre social. Renonçant un temps aux plans économiques, il mettra au point une méthode graphique de transcription de données statistiques qui se fera mondialement connaître sous le nom d'Isotype. Ce «langage visuel» devait permettre à l'homme de la rue de comprendre son environnement social et économique et d'ainsi s'auto-émanciper.

Ernst Toller passera cinq ans en prison et en sortira à l'âge de 30 ans. Il s'engagera pour le pacifisme et ses pièces et écrits connaîtront un succès international. Bien avant ses contemporains, il avait perçu les enjeux de la Révolution allemande: «Qui veut comprendre la faillite de 1933 doit connaître les événements des années 1918 et 1919 en Allemagne » ${ }^{(46)}$, écrira-t-il dans ses mémoires.

\section{Conclusion}

Malgré ses déclarations de satisfaction, Diederichs n’a, en réalité, pu éviter que les rencontres de Lauenstein ne ressemblent à une «tour de Babel ${ }^{(47)}$. Des lignes de fracture profondes parcouraient l'édifice dans tous les sens, entre les défenseurs d'une culture allemande "supérieure» et les tenants d'une culture intégrative, mais aussi entre les prophètes d'un renouveau spirituel et les réformateurs pragmatiques. Fractures que la République de Weimar sera bien en peine de réduire.

Plus marqué encore était le clivage entre les «anciens ", restés "à l’arrière», qui éludaient la dimension morale de la question de la guerre et semblaient ainsi s'arroger une rédemption sans jugement, et les «jeunes», revenus du front avec le sentiment d'avoir été floués, qui conféraient à cette expérience traumatisante une dimension éthique.

Cependant, à l'intérieur de cette jeune génération même, une autre divergence se dessine clairement à Lauenstein. Ne se retrouvant pas vraiment dans les jeunesses bündisch ${ }^{(48)}$ issues des mouvements de jeunesse, celles qui siéent à Eugen Diederichs

44 Voir les comptes rendus de la presse: «Zeugenaussage im Prozeß gegen Ernst Toller» et «Zeugenaussage im Prozeß gegen Otto Neurath» reproduits et commentés dans Mommsen/Schwentker (éd.), Max Weber. Zur Neuordnung Deutschlands (note 15), p. 485-495.

45 «[...] das ganze Gericht geriet eben in gute Laune, als ich die ganze Verdrehtheit dieser Lauensteiner Sache erzählte, und das nützt immer.» Lettre de Max Weber à Marianne Weber du 19 juillet 1919, reproduite dans Gerd Krumreich et Rainer Lepsuis (éd.), Max Weber: Briefe 1918-1920, vol. 2, Tübingen, Mohr Siebeck, 2012, p. 697-699, ici p. 699.

46 E. Toller, Eine Jugend in Deutschland (note 25), p. 237.

47 Lettre d'Eugen Diederichs à Max Weber du 22 juin 1917, HS.1995.0002, archives de la maison d'édition Eugen Diederichs, DLA.

48 Sur l'évolution ultérieure des jeunesses bündisch, voir Nicolas LE Moigne, "La "jeunesse ligueuse” (Bündische Jugend). Utopie et alternative politique dans l'Allemagne de Weimar», in: Olivier DARD et Étienne Desch a mps (éd.), Les relèves en Europe d'un après-guerre à l'autre. Racines, réseaux, projets et postérités, Bruxelles, Peter Lang, 2008, p. 249-266. 
et qui irritent Max Weber, certains jeunes intellectuels vont se politiser et s'engager dans la révolution, seule réponse possible à leur désir d'un véritable renversement de l'ordre ancien.

On les moquera alors de sobriquets, tels celui de Kaffeehausliteraten, parce que ces jeunes penseurs qui parlaient au nom des masses, n'appartenaient pas, pour autant, aux classes populaires. Cette autre fracture entre l'élite du Bildungsbürgertum et le monde ouvrier avait été totalement esquivée à Lauenstein. Le poète ouvrier Karl Bröger - selon ses propres termes le seul «inculte» de cette belle assemblée savante - ne s’y était pas senti à sa place. Par la suite, Diederichs lui permit de faire part de sa déception dans les pages du journal Die Tat. Selon Bröger, quatre-vingts personnes s'étaient rassemblées, chacune "un plan à la main", et elles s'étaient adonnées à de gentilles spéculations visant à modifier l'ordre existant au lieu d'envisager sa refondation radicale:

«Avec frénésie, chacun a contribué à tisser des toiles d'araignée de spéculations intellectuelles. Tous les angles et les recoins de la vieille salle d'armes ont fini par en être remplis. Partout, une grosse araignée mentale était à l'affût de tout ce qui bouge et vit. Si une idée vivante s'en venait à voleter à travers la pièce, elle finissait très vite par se prendre lamentablement dans ces filets ${ }^{(49)}$.

Et d'en conclure pour son compte: «Tu viens du peuple, tu appartiens à l'autre côté! »

\section{Résumé}

En 1917, l'éditeur Eugen Diederichs organisait deux séminaires au château Lauenstein de Thuringe. Des intellectuels de toutes tendances étaient conviés à débattre de l'avenir culturel et politique de l'Allemagne et à confronter leurs propositions de réforme. Le présent article retrace le déroulement de ces "rencontres culturelles de Lauenstein" et évoque les incidents qui les ont émaillées. Marquées par la figure dominante de Max Weber, elles ont mis au jour l'antagonisme des différents points de vue et incité certains jeunes participants à rompre avec l'ordre ancien. Le dramaturge Ernst Toller et l'économiste Otto Neurath s'engageront par la suite dans la Révolution de Novembre.

\section{Zusammenfassung}

1917 veranstaltete der Verleger Eugen Diederichs zwei Seminare auf der Burg Lauenstein in Thüringen. Eingeladene Intellektuelle jeder Couleur sollten über die kulturelle und politische Zukunft Deutschlands debattieren und praktische Reformvorschläge formulieren. Dieser Artikel schildert den Ablauf der "Lauensteiner Kulturtagungen“ und erwähnt die Zwischenfälle, die sie überschatteten. Geprägt durch die überragende Persönlichkeit Max Webers, haben diese Begegnungen die Unvereinbarkeit der Sichtweisen zu Tage gefördert und manch jungen Teilnehmer zum Bruch mit der alten Ordnung animiert. Daraufhin werden sich der Dramatiker Ernst Toller und der Nationalökonom Otto Neurath an der Novemberrevolution in Bayern beteiligen. 


\begin{abstract}
In 1917 the publisher Eugen Diederichs held two seminars at Lauenstein castle in Thuringia. Intellectuals of every color were invited to discuss the cultural and political future of Germany and exchange their views on proposed reforms. This article recounts how these "Lauensteiner Kulturtagungen" took place and the incidents which occurred here and there. Deeply influenced by Max Weber's outstanding personality, these encounters did highlight the antagonism between the different points of view and prompted younger participants to reject the old order. The dramatist Ernst Toller and the economist Otto Neurath were ultimately to get involved in the November Revolution in Bavaria.
\end{abstract}

